\title{
The Study on the MRTD Measurement System of Medical Infrared Thermal Imager Based on Sub-Pixel Edge Detection Algorithm
}

\author{
Cao Miao ${ }^{1}$, Cui Wenjie ${ }^{* 1}$ and Yao qinghua ${ }^{2}$ \\ ${ }^{1}$ Changchun University of Science and Technology, Jilin Changchun, 130022, \\ China \\ ${ }^{2}$ Changchun University Of Technology, Jilin Changchun, 130012, China \\ 79816540@qq.com
}

\begin{abstract}
MRTD (minimum resolvable temperature difference) is an important parameter to evaluate the infrared thermal imager. MRTD is not only related to the spatial resolution of the system, but also to many factors such as optical system, detector array and so on. The measurement technique and principle of the medical infrared thermal imager's MRTD is deeply analyzed, and the MRTD measurement model is established in this paper. The MRTD measurement system of infrared thermal imager based on CCD imaging technology is designed. CCD is used to carry on the interpretation of temperature difference in this system instead of the human eye, which can avoid the effect of some factors on the subjective image quality evaluation, such as the contrast, the human interference flaw and so on. The sub-pixel edge detection algorithm is used to deal with the four-bar target image, which can effectively suppress the interface of the random noise on four-bar target image. The experimental results show that the MRTD testing accuracy of the system can reach $0.055^{\circ} \mathrm{C}$.
\end{abstract}

Keywords: MRTD, the infrared thermal imager, $C C D$, image processing

\section{Introduction}

The infrared thermal imager is a kind of test equipment. It carries on the target material testing through the principle of infrared imaging, and detects the object that the human eyes can't identify. Non-contact manner is the main method for the infrared thermal imager to detect the infrared heat. The detection results can generate a certain image. Then after a rigorous calculation, image data is calculated, and this data is need to collect in the future by the staff members. This device can calculate the temperature of the target material, which is a sophisticated detection instrument in industrial detection. The collected temperature data will be qualified concretely, so that the heat generated in these areas can be identified by the staff, and then more specific analysis can be obtained. In recent years, the infrared thermal imager has been widely used. MRTD is an important parameter for evaluating the infrared thermal imager.

\section{The Establishment of MRTD Measurement Model}

\subsection{Traditional MRTD Measurement}

The traditional MRTD measurement method is the subjective method. The method uses the human eyes to interpret, and puts the four-bar target with specific space frequency and height-width ratio of 7:1 on a uniform background. The temperature difference of the target and the background is increased gradually from zero by the adjusting temperature blackbody. When the observer is able to distinguish the four-bar target image, the 
temperature difference between the target and the background is MRTD. The measurement principle is shown in Figure 1.

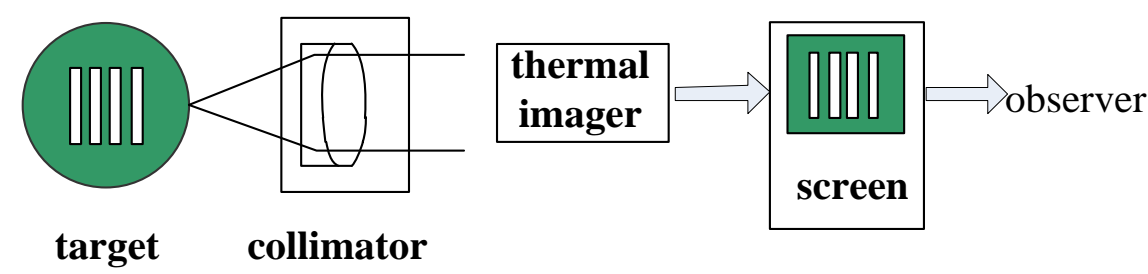

Figure 1. MRTD's Subjective Measurement Principle Diagram

In the process of measurement, the interpretation results are different because of the different image interpretation, or the different mental state of the same interpretation. When subjective measurement method is used to measure MRTD, the result is not stable, and the repeatability is poor. So the new technology is needed to form the objective judgment standard. In this paper, the results of previous studies are analyzed, and the MRTD measurement technology of the infrared thermal imager is designed based on CCD imaging technology.

\subsection{MRTD Measurement Model}

$\mathrm{CCD}$ is used to replace the human eyes to interpret the temperature difference based on the MRTD subjective measurement method. In consideration of the need of establishing an approximate infinity benchmark target when measuring MRTD, the collimator is used to simulate in this paper. The system composition is shown in Figure 2.

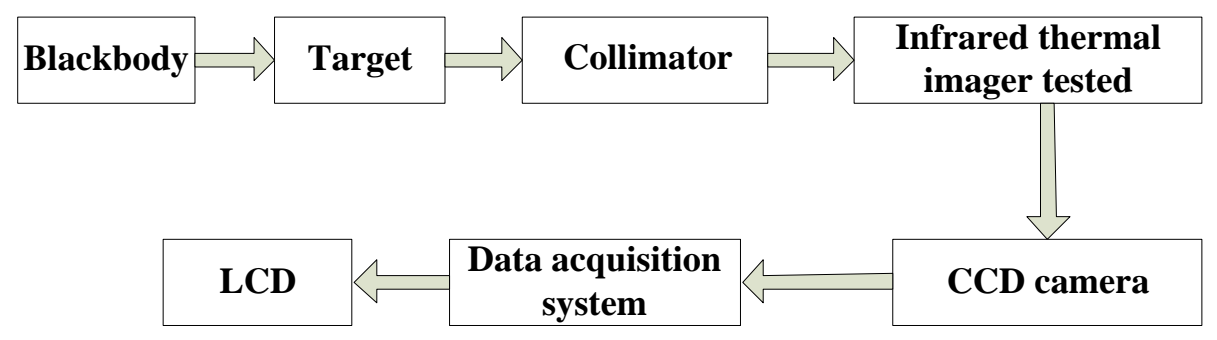

Figure 2. The Block Diagram of MRTD Measurement System

The target used in the MRTD temperature difference interpretation is a 4-bar target, and it is shown in Figure 3.

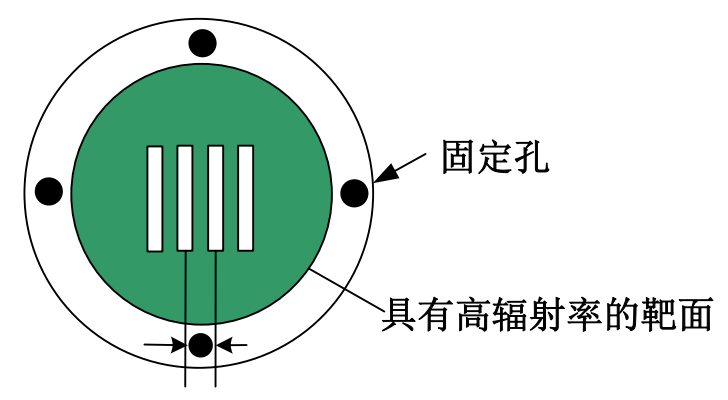

Figure 3. 4-Bar Target

The variable temperature target generators are composed of a surface source blackbody and a four-bar target, which provide a variable temperature target for the test system. The 
collimator provides an infinite and parallel beam, which provides a reference target for testing. The spatial frequency of the four-bar target is selected by $0.2 \mathrm{f}, 0.5 \mathrm{f}, 1 \mathrm{f}, 1.2 \mathrm{f}$ value ( $\mathrm{f}$ is $50 \%$ of the characteristic frequency of the infrared thermal imager being tested). The infrared target of the four-bar target gets through the infrared thermal imager being tested. It is received by the CCD sensor, which is collected in the computer by the image acquisition card and processed by the MATLAB.

By adjusting the temperature of the surface source blackbody, the target temperature is gradually above the background temperature.

In consideration of the temperature difference of hot rod, it is the temperature difference between $75 \%$ 0of each bar target and $75 \%$ of two bar targets, and it is observed, record the image and the temperature difference. The temperature difference until the cold bar appears is to lower continually, and the image and temperature are recorded differently. Repeat the above operation, record large amounts of data as training samples. The nonlinear mapping relationship between the input and output is obtained, which can be used to calculate the temperature difference of the hot bar and the cold bar.

In the measurement, it is called positive temperature difference ${ }^{\Delta T_{1}}$ when the target temperature is higher than the background temperature; it is called negative temperature

$\Delta T_{2}$ difference when the target temperature is lower than the background temperature. The average value of the absolute value is taken.

$$
\begin{aligned}
& \Delta T_{1}=T_{1}-T_{0} \\
& \Delta T_{2}=T_{2}-T_{0}
\end{aligned}
$$

Wherein, $\mathrm{T}_{0}$ is the equivalent environment temperature, $\mathrm{T}_{1}$ is the target temperature when the four-bar white striped pattern can be distinguished, $T_{2}$ is the target temperature when the four-bar black striped pattern can be distinguished.

The MRTD of the infrared thermal imager is:

$$
M R T D=K * \frac{\left|\Delta T_{1}\right|+\left|\Delta T_{2}\right|}{2}
$$

$\mathrm{K}$ is the system correction factor.

Put K, the positive and negative temperature difference into (3), the MRTD of the thermal imager tested can be obtained.

\section{The MRTD Measurement System of Infrared Thermal Imager Based on CCD Imaging Technology}

In this paper, a Newton type reflective collimator system is constructed, in which the off-axis parabolic mirror is the main body. It can provide infinity infrared target for the infrared thermal imager tested. The target is composed of the infrared reticle located at the focal point of the off-axis parabolic mirror and the high control-temperature-precision blackbody behind the reticle. The infrared thermal imager will display the image of the infinite far infrared target on its own screen. If there is a video signal output port, the video signal is captured through the video capture card and analyzed and processed by the computer to obtain the MRTD parameter. If there is no video signal output port, the infrared camera display is captured by the CCD. the camera's video signal is processed to obtain the relevant performance parameters. The structure of the system is shown in Figure 4.

The infrared collimator is composed of surface blackbody, target, off-axis parabolic mirror and body tube which has shielding mixed astigmatism, dust-proof function tube composition. The data processing system is composed of the computer and the corresponding board and the special software, which is the man-machine interactive medium for the completion of the parameter-test operation. 
In this system, the surface blackbody is designed and made with precision temperature measurement and control technology to meet the following performance indicators:

Differential temperature range: $-10^{\circ} \mathrm{C} \sim 50^{\circ} \mathrm{C}$;

Effective radiating area: $\geq \Phi 30 \mathrm{~mm}$;

Temperature resolution: $0.01{ }^{\circ} \mathrm{C}$;

Temperature control accuracy: $\pm 0.3{ }^{\circ} \mathrm{C}$;

Temperature uniformity: $0.1^{\circ} \mathrm{C}\left(\Delta \mathrm{T} \leq \pm 5^{\circ} \mathrm{C}, 2 / 3\right.$ of the central region);

Temperature stability: $\pm 0.01^{\circ} \mathrm{C} / \mathrm{h}$;

Effective emissivity: $\geq 0.95$.

The off-axis parabolic mirror is the main body of infrared collimator. According to the need of the function and accuracy, the focal length $\mathrm{f}=1500 \mathrm{~mm}$, the diameter $\mathrm{D}=\Phi 150 \mathrm{~mm}$, the spectral range: $8 \sim 14 \mu \mathrm{m}$.

The CCD of $\geq 1000 \times 1000$ resolution is needed in the CCD imaging system. So the Canadian Lumenera company's Lu175m camera is used.

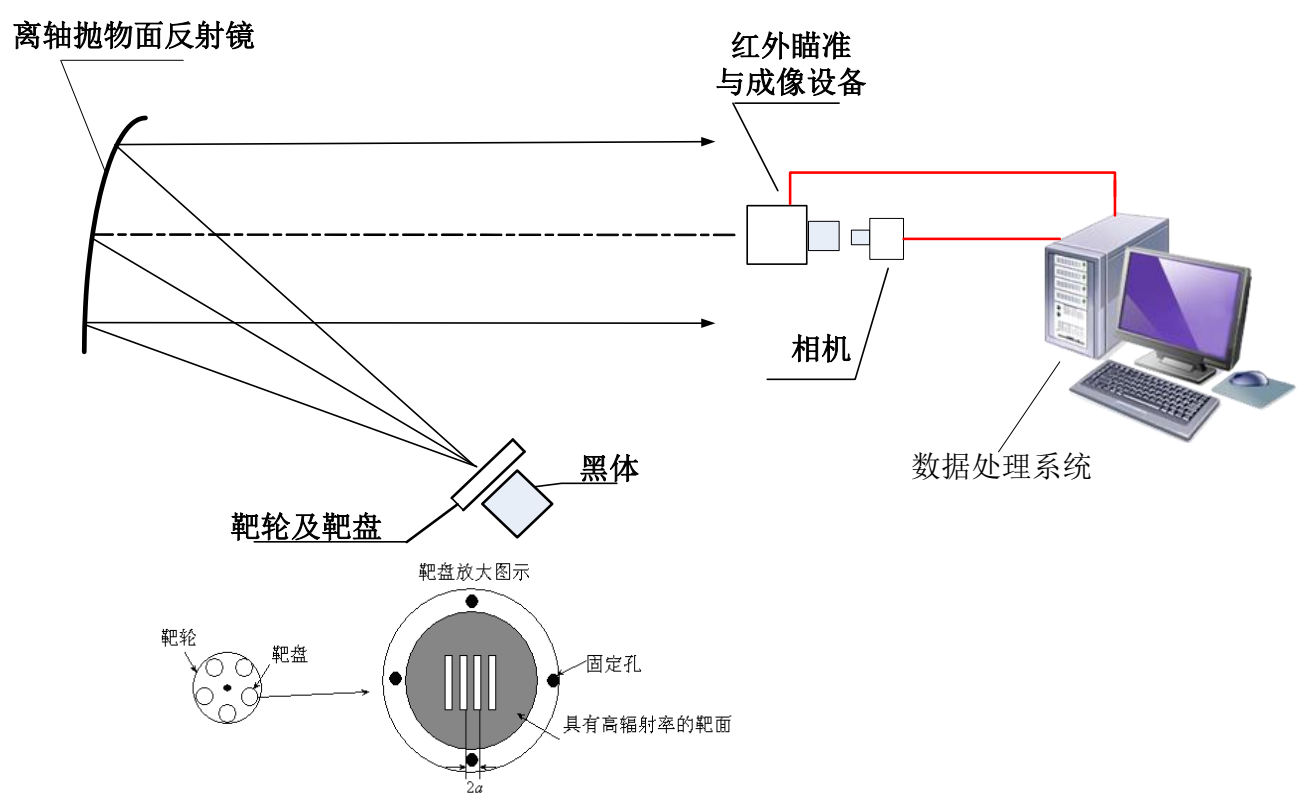

Figure 4. The MRTD Measurement System of Infrared Thermal Imager

\section{Four-Bar Target Image Processing}

When it is collected into the computer through the image acquisition card, the four-bar target image is a high noise image. The system uses the sub pixel edge detection algorithm to achieve the processing of the four-bar target image. The steps are as followed:

(1) The coarse positioning of the four-bar target image is carried out by using the conventional Sobel edge detection operator. The weighted difference of the adjacent point's gray around each pixel in the digital image $\{f(x, y)\}$ is studied, and the operator Sobel is obtained as follows: 


$$
\begin{aligned}
s(i, j) & =\left|\Delta_{x} f\right|+\left|\Delta_{y} f\right| \\
& =\left|\begin{array}{l}
(f(i-1, j-1)+2 f(i-1, j)+f(i-1, j+1))- \\
(f(i+1, j-1)+2 f(i+1, j)+f(i+1, j+1))
\end{array}\right| \\
& +\left|\begin{array}{l}
((f(i-1), j-1)+2 f(i, j-1)+f(i+1, j-1))- \\
(f(i-1, j+1)+2 f(i, j+1)+f(i+1, j+1))
\end{array}\right|
\end{aligned}
$$

The convolution operator is as followed:

$$
\Delta_{x} f=\left|\begin{array}{ccc}
-1 & 0 & 1 \\
-2 & 0 & 2 \\
-1 & 0 & 1
\end{array}\right|, \Delta_{y} f=\left|\begin{array}{ccc}
-1 & -2 & -1 \\
0 & 0 & 0 \\
1 & 2 & 1
\end{array}\right|
$$

Take appropriate threshold $\mathrm{TH}$, and judgment is as follows: for example, if $s(i, j)>T H,(i, j)$ is the step edge point, $\{s(i, j)\}$ is the edge image.

At the edge of the image, that is the extreme point. Its second order derivative is zero. So it takes a point in a small neighborhood on both sides of edge point, and calculate gradient direction value $\theta$ of each point in the small field. The formula is:

$$
\theta(i, j)=\cot \left(f_{y}^{\prime} / f_{x}^{\prime}\right)
$$

If the gradient magnitude of the neighborhood center is larger than the gradient magnitude of the two adjacent points along the gradient direction, this point is an edge point, and is set to 1; otherwise it is a non-edge point, and is set to 0. So an edge image single pixel is get.

(3)According to the procedure (1)(2), the Gaussian curve fitting can be carried out in the gradient direction. the Gauss curve expression is (7):

$$
y=\frac{1}{\sqrt{2 \pi \sigma}} \exp \left(\frac{-(x-\mu)^{2}}{2 \sigma^{2}}\right)
$$

On both sides of formula(7) are taken as the logarithm:

$$
\ln y=-\frac{(x-\mu)^{2}}{2 \sigma^{2}}+\ln \frac{1}{\sqrt{2 \pi \sigma}}
$$

Formula(8) is a quadratic curve, with a binary quadratic polynomial to fit close to the logarithm value of pixel gradient in the neighborhood of the edge point. The surface fitting function is as followed:

$$
f(x, y)=a_{0}+a_{1} x+a_{2} x^{2}+a_{3} y+a_{4} y^{2}+a_{5} x y
$$

In which $\mathrm{x}, \mathrm{y}$ is the coordinate value of pixel, $f(x, y)$ is the gradient logarithm value of pixel point $(x, y)$. The gray value of pixels near the edge of the field $3 \times 3$ is used and the least square method, the coefficient of the fitting function is calculated.

$$
\delta_{\min }^{2}=\sum_{x=i-1}^{x=i+1} \sum_{x=j-1}^{x=j+1}\left[f(x, y)-\left(a_{0}+a_{1} x+a_{2} x^{2}+a_{3} y+a_{4} y^{2}+a_{5} x y\right)\right]^{2}
$$

Replace $x$ and ${ }^{y}$ in the surface function (10) with $\rho \cos (\theta)$ and $\rho \sin (\theta)$ respectively, and get a quadratic curve equation. The maximum value is the exact coordinate of the edge point. The quadratic curve equation is as followed: 


$$
\rho=-\frac{a_{1} \cos (\theta)+a_{3} \sin (\theta)}{2\left[a_{2} \cos ^{2}(\theta)+a_{5} \cos (\theta) \sin (\theta)+a_{4} \sin ^{2}(\theta)\right]}
$$

The coordinate of sub pixel edge point can be obtained:

$$
x=\rho_{g} \cos (\theta) \quad y=\rho_{g} \sin (\theta)
$$

In addition, with CCD instead of human eyes to observe and judge, in order to effectively suppress the interference of some random noise, the multi-frame image can be dealt with average processing to reduce the impact of noise on the judgment. By the experience, it is more reasonable to take 6 11 images for averaging. This paper takes an average of 9 images, and the processed image is shown in Figure 5.

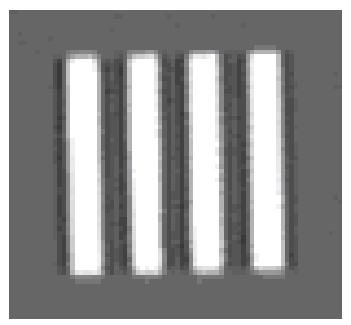

Figure 5. The Processed Image of Four-Bar Target

\section{Experimental Results and Conclusions}

With the traditional measuring method and CCD interpretation method respectively to measure the MRTD of the thermal infrared imager tested, 10 inferior precision experiments are carried out. The experimental data table is shown in Table 2.

Table 2. The Experimental Data Table

\begin{tabular}{ccccccc}
\hline \multirow{2}{*}{ Time } & \multicolumn{3}{c}{ traditional measuring method $\left({ }^{\circ} \mathrm{C}\right)$} & \multicolumn{3}{c}{ CCD interpretation method $\left({ }^{\circ} \mathrm{C}\right)$} \\
\cline { 2 - 7 } & +MRTD & - MRTD & MRTD & +MRTD & -MRTD & MRTD \\
\hline 1 & -0.018 & -0.121 & -0.062 & -0.013 & -0.136 & -0.052 \\
2 & -0.018 & -0.125 & -0.065 & -0.013 & -0.138 & -0.053 \\
3 & -0.019 & -0.117 & -0.062 & -0.011 & -0.136 & -0.052 \\
4 & -0.029 & -0.122 & -0.058 & -0.011 & -0.138 & -0.054 \\
5 & -0.018 & -0.124 & -0.066 & -0.012 & -0.135 & -0.053 \\
6 & -0.023 & -0.122 & -0.057 & -0.011 & -0.133 & -0.051 \\
7 & -0.027 & -0.116 & -0.058 & -0.009 & -0.135 & -0.054 \\
8 & -0.017 & -0.121 & -0.066 & -0.013 & -0.131 & -0.050 \\
9 & -0.017 & -0.129 & -0.065 & -0.013 & -0.135 & -0.052 \\
10 & -0.030 & -0.129 & -0.060 & -0.012 & -0.137 & -0.050 \\
\hline mean value & 0.0215 & 0.1241 & -0.0618 & 0.022 & 0.135 & -0.0514 \\
\hline$\square$ & 0.006 & 0.004 & 0.038 & 0.00167 & 0.0019 & 0.0015 \\
\hline
\end{tabular}

From Table 2, comparing the MRTD value measured by the subjective measurement method with that of CCD interpretation method measured, there are deviations in the mean value and standard deviation. As can be seen from the experimental data, CCD interpretation is relatively stable, and has a good reproducibility. 


\section{References}

[1] R. Rao, "Equivalence of MTF of a turbid medium and radiative transfer field", Chinese Optics Letters, vol. 10, no. 2, (2012), 020101.

[2] L. M. Biberman, "Electro-Optical Imaging System Performance and Modeling, Ch.12", SPIE, Bellingham, WA, (2000).

[3] R. Rao, "Equivalence of MTF of a turbid medium and radiative transfer field", Chinese Optics Letters, vol. 10, no. 2, (2012), 020101.

[4] S. Wen, D. bin and L. Jian, "Multiresolution distance map based small target detection in infrared image", Acta Electronica Sinica, vol. 30, no. 1, (2002), pp. $42-45$.

[5] R. L. Haney, "Principal component analysis for enhancement of infrared spectra monitoring", (2011).

[6] S. Q. Kun, "I mprove Fuzzy C - means Clustering Algorithm", Harbin University of Science and Technology, vol. 12, no. 4, (2007).

[7] J. Y. Shu, "Researchon Application of Analog-probefor Measuring Center", Harbin University of Science and Technology, vol. 14, no. 4, (2009), pp. 121-124.

[8] B. T. Ting, "Image Edge Detection Based on Wavelet Transform and Canny Operator", Harbin University of Science and Technology, vol. 15, no. 1, (2010). 
International Journal of Multimedia and Ubiquitous Engineering

Vol.11, No.7 (2016) 